\title{
Social-cognitive barriers to ethical authorship
}

\author{
Jordan R. Schoenherr ${ }^{1,2 *}$ \\ ${ }^{1}$ Department of Psychology, Carleton University, Ottawa, ON, Canada, ${ }^{2}$ Department of Innovation in Medical Education, \\ University of Ottawa, Ottawa, ON, Canada
}

Keywords: research misconduct, research integrity, inappropriate authorship, source credibility, applied ethics

\section{Introduction}

The apparent increase in research misconduct in the scientific literature has caused considerable alarm in both the biomedical (Benos et al., 2005; Smith, 2006) and psychological research communities (Stroebe et al., 2012). An understanding of research misconduct must be informed by the recognition that the norms of science might be quite general (e.g., Merton, 1942; Bronowski, 1965), ambiguous (Cournand and Meyer, 1976), or even contradictory (e.g., Mitroff, 1974; Ziman, 2000), leading to possible disagreements in terms of what constitutes misconduct within a research community (Fields and Price, 1993; Berk et al., 2000; Al-Marzouki et al., 2005). Considerable insight can be gained from research on behavioral ethics (e.g., Bazerman and Tenbrunsel, 2011; Ariely, 2012; Greene, 2013). Using inappropriate authorship practices as an illustrative example, I consider the role of social-cognitive mechanisms in research misconduct while also suggesting preventative measures.

\section{OPEN ACCESS}

Edited by:

Shahar Ayal,

Interdisciplinary Center Herzliya, Israel

Reviewed by:

Guy Hochman,

Duke University, USA

Andrea Pittarello,

Ben-Gurion University of the Negev,

Israel

${ }^{*}$ Correspondence:

Jordan R. Schoenherr, jordan.schoenherr@carleton.ca

Specialty section: This article was submitted to

Cognitive Science, a section of the journal

Frontiers in Psychology

Received: 05 March 2015 Accepted: 13 June 2015

Published: 21 July 2015

Citation:

Schoenherr JR (2015) Social-cognitive barriers to ethical authorship.

Front. Psychol. 6:877. doi: 10.3389/fpsyg.2015.00877

\section{Prevalence of Research Misconduct}

Widespread interest in dishonesty in research began comparatively recently in the history of the sciences (e.g., Broad and Wade, 1982; Steneck, 1999) although there was an early recognition that misconduct was a feature of scientific research (Babbage, 1830). Though a definitive set of forms of misconduct has yet to be identified, fabrication, falsification, and plagiarism (FFP) are generally cited as clear violations of scientific norms. In a review of studies of FFP, Steneck (2006) estimated that its occurrence rate fell within a range of 1.0 and $0.001 \%$ (for recent support, see Fanelli, 2009). $\mathrm{He}$ further suggested that research practices reflect a normal distribution, with FFP representing outlying behaviors. More ambiguous behaviors, or questionable research practices (QRP), have a much higher rate of occurrence, with Steneck suggesting that they constitute $10-50 \%$ of all research practices. QRPs represent an interesting form of misconduct in that they apparently reflect a feature of normal science (De Vries et al., 2006) thereby suggesting that they might reflect the socialcognitive processes underlying the dishonest behaviors of people more generally (e.g., Bazerman and Tenbrunsel, 2011; Ariely, 2012).

Inappropriate authorship practices are a prevalent form of QRP. For instance, they can represent a failure to recognize an original contribution to research (ghost authorship) or a misattribution of the research to those who have not contributed (gift authorship). The prevalence of inappropriate authorship practices is reflected in studies conducted by Flanagin et al. (1998) and Wislar et al. (2011) wherein they observed a decrease in the prevalence of ghost authorship from 11.5 to $7.9 \%$ between 1996 and 2008. In contrast, the number of articles affected only by gift authorship remained relative constant with a non-significant decrease from 19.3 to $17.6 \%$ during the same period (for similar findings, see Mowatt et al., 2002; Mirzazadeh et al., 2011; cf. Stretton, 2014). Accounting for the stability and change of inappropriate authorship practices represents an important task for applied ethics as the assignment of 
credit can lead to stratification within the scientific community (e.g., Cole and Cole, 1973).

\section{The Social Cognition of Credit and Credibility}

Early commentators attributed research misconduct to a range of factors including publication pressure, competition, and psychopathy (Chubin, 1985; cf. Braxton and Bayer, 1994). However, the prevalence of QRP suggests that more general social-cognitive mechanisms can account for research misconduct. Analyses of cases of misconduct have suggested a number of contributing factors (for a review, see Davis et al., 2007). Here I will consider how inappropriate authorship practices can be understood in terms of influence of social conventions and conformity, the reciprocity norms of exchange systems, as well as role schemata and status.

\section{Social Conventions and Conformity Bias}

The social conventions and ethical norms of science are evidenced in its cultural, structural, and organizational systems (Davis, 2003). Empirical support for the role of social conventions in judgements of ethical conduct comes from a number of sources. Kohlberg (1976) outlines a model with three stages of moral reasoning. A preconventional stage of moral reasoning defined by self-interest is contrasted against a subsequent stage of conventional moral reasoning wherein social norms of the group or society are used to judge behavior. While an additional post-conventional stage relies on the use of ethical principles, Kohlberg found that few individuals achieve this stage of reasoning (cf. Rest et al., 1999). Even when morals can be clearly identified, conventions play an important role in social interactions (Turiel, 2002) with conformity biases maintaining cultural norms (e.g., Whiten et al., 2005; Efferson et al., 2008). Experimental evidence also suggests that dishonest behaviors increase when in-group members are observed to engage in these behaviors (Gino et al., 2009).

Studies of academic misconduct have also demonstrated the influence of conventions and conformity, in terms of peer influence on cheating. In their study, McCabe and Treviño (1997) found that peer behavior and fraternity/sorority membership were positively related to the occurrence of misconduct, whereas perceived peer disapproval was negatively related to the occurrence of misconduct (see also, McCabe et al., 2001). Social conventions additionally offer an explanation for the difficulty in implementing successful ethics training programs, with disciplinary and departmental values being associated with researcher behavior (e.g., Anderson et al., 1994) and regression from post-conventional reasoning to conventional reasoning (Rennie and Rudland, 2002; Hren et al., 2011).

\section{Social Organization and Reciprocal Exchange}

The nature and prevalence of dishonesty can also be understood in terms of the norms of social exchange systems (e.g., Fiske, 1991). Fiske (1991) considers four kinds of exchange systems that differ in terms of the commensurability of the objects in the reciprocal exchange relationship (equality matching; communal sharing; market pricing; and authority ranking). These systems will in turn determine what is seen as honest and dishonest behavior. For instance, a researchers' contributions to a research project (e.g., theory, data collection, statistics) might be deemed unique and incommensurable, making judgments of proportion of credit arbitrary (communal sharing) or exceedingly difficult (equality matching). Researchers might instead assume that contributions can be differentiated and are quantifiable in terms of an absolute value that can be used to assign a proportion of authorship credit and responsibility (market pricing). Rightly or wrongly, this exchange norm appears to underscore the belief that the order of authorship reflects the proportion of contribution a researcher has made to a study (e.g., ICJME, 2005/2008). Finally, researchers might assume that authority should be the primary determinant of the assignment of credit (authority ranking), something that I will return to the next section.

Scientific research has been defined as an exchange system by a number of authors. Hagstrom (1982) suggested that a research article can be viewed as analogous to a gift whereas Street et al. (2010) have noted that "journal articles are valuable intellectual property," (p. 1458). These observations as well as others suggest that reciprocity can exert considerable influence on our judgements (Gouldner, 1960; Fiske, 1991). In terms of authorship, credit might be given due to the need for reciprocity by junior researchers receiving funding or advice from senior researchers. Authorship deals, or "mutual support authorships," wherein researchers include names of authors so as to have their name included on a project, also explicitly reflect an overt reciprocity strategy (Claxton, 2005; Louis et al., 2008). In addition to overt pressure, "lab chiefs" might be assigned undue credit as a result of researchers receiving career advice and financial support thereby enabling the research process while not directly contributing to intellectual content of a specific publication (Broad and Wade, 1982; Claxton, 2005; Street et al., 2010). Similarly, the provision of sponsorship might be perceived as sufficient grounds for receiving authorship (Louis et al., 2008). Both of these behaviors might be best understood in terms of the halo effect (Thorndike, 1920; Nisbett and Wilson, 1977) wherein participants overgeneralize from one attribute to the individual as a whole (see also, Harvey et al., 2010).

\section{Source Credibility, Status, and Role Schemata}

Due to the need to allocate limited attention, researchers must identify a subset of individuals that appear to provide credible information (Thorngate et al., 2011). Source credibility exerts considerable influence in the formation and change of attitudes (e.g., Petty et al., 1997). Thus, the contributions of researchers who are deemed to have greater credibility a priori might not be judged as critically as those with less credibility. Supporting this, studies that manipulate power (e.g., Guinote, 2013) have demonstrated that those in comparatively powerless position have reduced attention and short-term memory resources due to a need to respond to those in positions of power. In comparison, those in powerful positions are more likely to engage in confirmation bias in the pursuit of their goals. Collaborations between senior and junior researchers will likely be influenced 
by these situational factors (e.g., Sullivan and Ogloff, 1998) making it harder for junior members to assess the contributions of senior authors. Gift authorship can also be understood as an instance of a desire to confer credibility onto a research project. Peters and Ceci (1982) demonstrated this influence in a quasi-experiment wherein journal articles previously published by prestigious authors were resubmitted with fictitious nonprestigious names. When submitted with non-prestigious names, the majority of referees rejected these previously accepted articles.

The effects of source credibility can also be understood in terms of status assigned to social roles (e.g., Merton, 1968; Azoulay et al., 2014). Role schemata contain information pertaining to behaviors and obligations associated with a given role in a particular social context, thereby influencing the behavior and judgments of self and others. Historically, Shapin (1989) has noted that despite significant intellectual contributions to the design and conduct of experiments, technicians were not deemed to warrant authorship. As noted above, lab chiefs also appear to be awarded undue credit (Broad and Wade, 1982) and this might be attributed to perceived differences in credibility. If students and other personnel associated with a research project are believed to have a "supporting" role, their contributions might not be attributed to them. Rather, they might need to be legitimated by credible others in order for them to be accepted within a research community. More generally, authority ranking exchange systems assume that those in positions of authority are deemed to warrant more resources (Fiske, 1991). This would manifest itself as being awarded a disproportionate amount of credit. However, role schemata can also benefit those perceived to be in a subordinate position. As Zuckerman (1968) observed, Nobel laureates often appear to have awarded greater authorship credit to less prestigious collaborators. Moreover, those with higher status have also been found to express more favorable attitudes toward preserving the ethical norms of their discipline (e.g., Braxton and Bayer, 1994).

\section{Conclusions}

If inappropriate authorship practices can be accounted for by general social-cognitive processes, then an ameliorative program

\section{References}

Al-Marzouki, S., Roberts, I., Marshall, T., and Evans, S. (2005). The effect of scientific misconduct on the results of clinical trials: a delphi study. Contemp. Clin. Trials 26, 331-337. doi: 10.1016/j.cct.2005. 01.011

Anderson, M. S., Louis, K. S., and Earle, J. (1994). Disciplinary and departmental effects on observations of faculty and graduate student misconduct. J. High. Educ. 65, 331-350.

Ariely, D. (2012). The Honest Truth about Dishonesty: How We Lie to Everyone, Especially Ourselves. New York, NY: HarperCollins.

Azoulay, P., Stuart, T., and Wang, Y. (2014). Matthew: effect or fable? Manage. Sci. 60, 92-109. doi: 10.1287/mnsc.2013.1755 at least appears possible in principle. In opposition to these efforts, ethics training programs developed in an applied context have not always been successful (e.g., Brown and Kalichman, 1998; Fisher et al., 2009). Such failures likely stem from an ethical "fudge factor," a failure to attend to ethical norms on a moment-to-moment basis, and the observation of dishonest behavior of peers (e.g., Bazerman and Tenbrunsel, 2011; Ariely, 2012; Greene, 2013). Indeed, rather than engaging in an explicit reasoning process (Kohlberg, 1976; Rest et al., 1999) our responses to ethical dilemmas often appear to be automatic (Haidt, 2007) and are susceptible to loss framing and time pressure (e.g., Kern and Chugh, 2009). Together with selfdeception and justifications (Tenbrunsel and Messick, 2004; Shalvi et al., 2011), ethical facets of authorship decisions might become less salient. Reciprocity norms, along with the "publish or perish" framing of contemporary academic publishing, would certainly support these behaviors. These enablers must be acknowledged and addressed if we hope to reduce ghost and gift authorship.

Having recognized the influence of social context and automaticity, three general proposal appear to offer promise to reduce the prevalence of unethical behaviors. First, we must ensure that researchers are aware of the ethical standard and norms of authorship within their research community and that co-authors discuss expectations and roles throughout the research process. Standards such as those provided by the ICJME (2005/2008) are useful points of reference for the assignment of authorship/contributorship. Second, by continually priming these norms with ongoing discussions at departmental and disciplinary levels, we are likely to obtain similar reductions in dishonest behavior as those observed in laboratory studies (Mazar et al., 2008). Finally, to disincentivize dishonest behavior stemming from a "publish or perish" academic culture, we must consider adopting criterion for hiring, promotion, and funding decisions based on the quality of a restricted number of publications rather than the total number of publications produced by an individual.

\section{Funding}

This research was supported by funding from the Ottawa Health Research Institute. 
Braxton, J. M., and Bayer, A. E. (1994). Perceptions of research misconduct and an analysis of their correlates. J. High. Educ. 65, 351-372. doi: 10.2307/2943972

Broad, W. J., and Wade, N. (1982). Betrayers of the Truth. New York, NY: Simon and Schuster.

Bronowski, J. (1965). Science and Human Values, Rev Edn. New York, NY: Harper Torchbooks.

Brown, S., and Kalichman, M. W. (1998). Effects of training in the responsible conduct of research. Sci. Eng. Ethics 4, 487-498. doi: 10.1007/s11948-9980041-y

Chubin, D. E. (1985). Misconduct in research: an issue of science policy and practice. Minerva 23, 175-202. doi: 10.1007/BF01099941

Claxton, L. D. (2005). Scientific authorship. Part 2. History, recurring issues, practices, and guidelines. Mutat. Res. 589, 31-45. doi: 10.1016/j.mrrev.2004.07.002

Cole, J. R., and Cole, S. (1973). Social Stratification in Science. Chicago, IL: University of Chicago Press.

Cournand, A., and Meyer, M. (1976). The Scientist's Code. Minerva 14, 79-96. doi: 10.1007/BF01096215

Davis, M. S. (2003). The role of culture in research misconduct. Account. Res. 10, 189-201. doi: 10.1080/714906092

Davis, M. S., Riske-Morris, M., and Diaz, S. R. (2007). Causal factors implicated in research misconduct: evidence from ORI case files. Sci. Eng. Ethics 13, 395-414. doi: 10.1007/s11948-007-9045-2

De Vries, R., Anderson, M. S., and Martinson, B. C. (2006). Normal misbehavior: scientists talk about the ethics of research. J. Empir. Res. Hum. Res. Ethics 1, 43-50. doi: 10.1525/jer.2006.1.1.43

Efferson, C., Lalive, R., Richerson, P. J., McElreath, R., and Lubell, M. (2008). Conformists and mavericks: the empirics of frequencydependent cultural transmission. Evol. Hum. Behav. 29, 56-64. doi: 10.1016/j.evolhumbehav.2007.08.003

Fanelli, D. (2009). How many scientists fabricate and falsify research? A systematic review and meta-analysis of survey data. PLoS ONE 4:e5738. doi: 10.1371/journal.pone.0005738

Fields, K. L., and Price, A. R. (1993). Problems in research integrity arising from misconceptions about the ownership of research. Acad. Med. 68, S60-S64. doi: 10.1097/00001888-199309000-00036

Fisher, C. B., Fried, A. L., and Feldman, L. G. (2009). Graduate socialization in the responsible conduct of research: a national survey on the research ethics training experiences of psychology doctoral students. Ethics Behav. 19, 496-518. doi: 10.1080/10508420903275283

Fiske, A. P. (1991). Structures of Social Life: The Four Elementary Forms of Human Relations. New York, NY: Free Press.

Flanagin, A. Carey, L. A., Fontanarosa, P. B., Phillips, S. G., Pace, B. P., Lundberg, G. D., et al. (1998). Prevalence of articles with honorary authors and ghost authors in peer-reviewed medical journals. JAMA 280, 222-224. doi: 10.1001/jama.280.3.222

Gino, F., Ayal, S., and Ariely, D. (2009). Contagion and differentiation in unethical behavior: the effect of one bad apple on the barrel. Psychol. Sci. 20, 393-398. doi: $10.1111 / j .1467-9280.2009 .02306 . x$

Gouldner, A. W. (1960). The norm of reciprocity: a preliminary statement. Am. Sociol. Rev. 25, 161-178. doi: 10.2307/2092623

Greene, J. (2013). Moral Tribes: Emotion, Reason, and the Gap Between Us and Them. New York, NY: The Penguin Press.

Guinote, A. (2013). "Social power and cognition," in The Oxford Handbook of Social Cognition, ed D. E. Carlston ( New York, NY: Oxford University Press), 575-587. doi: 10.1093/oxfordhb/9780199730018.013.0028

Hagstrom, W. O. (1982). "Gift giving as an organizing principle in science," in Science in Context: Readings in the Sociology of Science, eds B. Barnes and D. O. Edge ( Cambridge: MIT Press), 21-34.

Haidt, J. (2007). The new synthesis in moral psychology. Science 316, 998-1002. doi: $10.1126 /$ science. 1137651

Harvey, A., Kirk, U., Denfield, G. H., and Read, P. (2010). Monetary favors and their influence on neural responses and revealed preference. J. Neurosci. 30, 9597-9602. doi: 10.1523/JNEUROSCI.1086-10.2010

Hren, D., Marušić, M., and Marušić., A. (2011). Regression of moral reasoning during medical education: combined design study to evaluate the effect of clinical study years. PLoS ONE, 6:e17406. doi: 10.1371/journal.pone. 0017406
International Committee of Medical Journal Editors (ICJME). (2005/2008). Uniform Requirements for Manuscripts Submitted to Biomedical Journals: Writing and Editing for Biomedical Publication. Available online at: www.icmje. org on November, 2008.

Kern, M., and Chugh, D. (2009). Bounded ethicality: the perils of loss framing. Psychol. Sci. 20, 378-384. doi: 10.1111/j.1467-9280.2009.02296.x

Kohlberg, L. (1976). "Moral stages and moralization: the cognitive-development approach," in Moral Development and Behavior: Theory, Research and Social Issues, ed T. Lickona (New York, NY: Holt, Rinehart and Winston), $31-53$.

Louis, K. S., Holdsworth, J. M., Anderson, M. S., and Campbell, E. G. (2008). Everyday ethics in research: translating authorship guidelines into practice in the bench sciences. J. Higher Educ. 79, 88-112. doi: 10.1353/jhe.20 08.0002

Mazar, N., Amir, O., and Ariely, D. (2008). The dishonesty of honest people: a theory of self-concept maintenance. J. Mark. Res. 45, 633-644. doi: $10.1509 /$ jmkr.45.6.633

McCabe, D. L., and Treviño, L. K. (1997). Individual and contextual influences on academic dishonesty: a multicampus investigation. Res. High. Educ. 38, 379-396.

McCabe, D. L., Treviño, L. K., and Butterfield, K. D. (2001). Cheating in academic institutions: a decade of research. Ethics Behav. 11, 219. doi: $10.1207 /$ S15327019EB1103_2

Merton, R. K. (1942). "The normative structure of science," in The Sociology of Science: Theoretical and Empirical Investigations, ed R. K. Merton (Chicago, IL: University of Chicago Press), 267-278.

Merton, R. K. (1968). The Matthew effect in science. Science 159, 56-63. doi: 10.1126/science.159.3810.56

Mirzazadeh, A., Navadeh, S., Rokni, M. B., and Farhangniya, M. (2011). The prevalence of honorary and ghost authorships in Iranian biomedical journals and its associated factors. Iran J. Public Health 40, 15-21. Available online at: http://www.ncbi.nlm.nih.gov/pmc/articles/PMC3481730/

Mitroff, I. I. (1974). Norms and counter-norms in a select group of the Apollo moon scientists: a case study of the ambivalence of scientists. Am. Sociol. Rev. 39, 579-595. doi: 10.2307/2094423

Mowatt, G., Shirran, L., Grimshaw, J. J. M., Rennie, D., Flanagin, A., Yank, V. et al. (2002). Prevalence of honorary and ghost authorship in Cochrane reviews. J. Am. Med. Assoc. 287, 2769-2771. doi: 10.1001/jama.287. 21.2769

Nisbett, R. E., and Wilson, T. D. (1977). The halo effect: Evidence for unconscious alteration of judgments. J. Pers. Soc. Psychol. 35, 250-256. doi: 10.1037/00223514.35.4.250

Peters, D., and Ceci, S. (1982). Peer-review practices of psychological journals: the fate of published articles, submitted again. Behav. Brain Sci. 5, 187-195. doi: 10.1017/S0140525X00011183

Petty, R. E., Wegener, D. T., and Fabrigar, L. R. (1997). Attitudes and attitude change. Annu. Rev. Psychol. 48, 609-647. doi: 10.1146/annurev.psych. 48.1.609

Rennie, S. C., and Rudland, J. R. (2002). Differences in medical students' attitudes to academic misconduct and reported behaviour across the years: a questionnaire study. J. Med. Ethics 29, 97-102. doi: 10.1136/jme. 29.2.97

Rest, J., Narvaez, D., Bebeau, M., and Thoma, S. (1999). A Neo-Kohlbergian approach: the DIT and schema theory. Educ. Psychol. Rev. 11, 291-324. doi: 10.1023/A:1022053215271

Shalvi, S., Dana, J., Handgraaf, M. J. J., and De Dreu, C. K. W. (2011). Justified ethicality: Observing desired counterfactuals modifies ethical perceptions and behavior. Organ. Behav. Hum. Decis. Process. 115, 181-190. doi: 10.1016/j.obhdp.2011.02.001

Shapin, S. (1989). The invisible technician. Am. Sci. 77, 554-563.

Smith, R. (2006). Research misconduct: the poisoning of the well. J. R. Soc. Med. 99, 232-237. doi: $10.1258 /$ jrsm.99.5.232

Steneck, N. (1999). Confronting misconduct in science in the 1980s and 1990s: What has and has not been accomplished? Sci. Eng. Ethics 5, 161-175. doi: 10.1007/s11948-999-0005-x

Steneck, N. (2006). Fostering integrity in research: definitions, current knowledge, and future directions. Sci. Eng. Ethics 12, 53-74. doi: 10.1007/s11948-0060006-y 
Street, J. M., Rogers, W. A., Israel, M., and Braunack-Mayer, A. J. (2010). Credit where credit is due? Regulation, research integrity and the attribution of authorship in the health sciences. Soc. Sci. Med. 70, 1458-1465. doi: 10.1016/j.socscimed.2010.01.013

Stretton, S. (2014). Systematic review on the primary and secondary reporting of the prevalence of ghostwriting in the medical literature. BMJ Open 4:e004777. doi: 10.1136/bmjopen-2013-004777

Stroebe, W., Postmes, T., and Spears, R. (2012). Scientific misconduct and the myth of self-correction in science. Perspect. Psychol. Sci. 7, 670-688. doi: $10.1177 / 1745691612460687$

Sullivan, L. E., and Ogloff, J. R. P. (1998). Appropriate supervisor-graduate student relationships. Ethics Behav. 8, 229-248. doi: 10.1207/s15327019eb0803_4

Tenbrunsel, A. E., and Messick, D. M. (2004). Ethical fading: the role of self-deception in unethical behavior. Soc. Justice Res., 17, 223-236. doi: 10.1023/B:SORE.0000027411.35832.53

Thorndike, E. L. (1920). A constant error in psychological ratings. J. Appl. Psychol. 4, 25-29.

Thorngate, W., Liu, J., and Chowbhury, W. (2011). The competition for attention and the evolution of science. J. Artif. Soc. Soc. Simul. 14, 1-6. Available online at: http://jasss.soc.surrey.ac.uk/14/4/17.html

Turiel, E. (2002). The Culture of Morality: Social Development, Context, and Conflict. New York, NY: Cambridge University Press. doi: 10.1017/CBO9780511613500
Whiten, A., Horner, V., and de Waal, F. B. M. (2005). Conformity to cultural norms of tool use in chimpanzees. Nature 437, 737-740. doi: $10.1038 /$ nature 04047

Wislar, J. S., Flanagin, A., Fontanarosa, P. B., and DeAngelis, C. D. (2011) Honorary and ghost authorship in high impact biomedical journals: a cross-sectional survey. Br. Med. J. 343:d6128. doi: 10.1136/bmj. d6128

Ziman, J. (2000). Real Science: What it is and What it Means. Cambridge: Cambridge University Press.

Zuckerman, H. A. (1968). Patterns of name ordering among authors of scientific papers: a study of social symbolism and its ambiguity. Am. J. Sociol. 74, 276-291. doi: $10.1086 / 224641$

Conflict of Interest Statement: The authors declare that the research was conducted in the absence of any commercial or financial relationships that could be construed as a potential conflict of interest.

Copyright (c) 2015 Schoenherr. This is an open-access article distributed under the terms of the Creative Commons Attribution License (CC BY). The use, distribution or reproduction in other forums is permitted, provided the original author(s) or licensor are credited and that the original publication in this journal is cited, in accordance with accepted academic practice. No use, distribution or reproduction is permitted which does not comply with these terms. 